ten täglich. Vielleicht brauchen wir sogar mehr. Ich habe bei meinen über 60-jährigen Patienten zu 82 Prozent einen Vitamin-DMangel im Winter festgestellt, 48 Prozent haben auch im Sommer ein Vitamin-D-Defizit. Die Problematik scheint sogar zuzunehmen, ohne dass wir dies genau erklären können. Wir wissen, dass Altershaut vergleichsweise weniger UV-B-Licht aufnimmt. Sonnencremes mit hohen Lichtschutzfaktoren verhindern ebenfalls, dass UV-B-Licht eindringt. Zudem bewegt sich die Bevölkerung insgesamt immer weniger im Freien. Wir müssen also den Vitamin-D-Mangel sehr ernst nehmen. Die Blutkonzentration sollte mindestens $70 \mathrm{nmol} / \mathrm{l}$ beziehungsweise 27 bis $40 \mathrm{ng} / \mathrm{ml}$ betragen. Wenn eine Erkrankung der Nebenschilddrüse mit Hyperkalziämie vorliegt, darf natürlich kein Vitamin D verabreicht werden.

? Wenig gesprochen wird auch über die Bedeutung einer ausreichenden Proteinzufuhr. Die rein pflanzliche Ernährung erscheint immer mehr Menschen gesünder als Fleisch und Wurst ...

Semler: Hauptproblem sind die Veganer, die jegliches tierisches Eiweiß ablehnen. Das wenige pflanzliche Protein reicht jedoch nicht aus, weshalb diese Menschen wegen der Auswirkungen auf die Muskulatur zur Gruppe der Osteoporosegefährdeten zählen. Zuviel tierisches Eiweiß ist aber auch nicht gut. Wir brauchen eine eher basische Kost, denn dann geht weniger Kalzium über die Nieren verloren.

? Eines der Hauptprobleme für viele Menschen ist der chronische Mangel an Bewegung. Wie kann man Bewegungsmuffel zu mehr körperlicher Aktivität motivieren?

Semler: Das ist sehr schwierig. Wichtig ist es zu versuchen, Bewegung in den Alltag einzubauen, etwa indem man Lifte und Rolltreppen meidet und statt dessen Treppen steigt. Mancher kauft sich einen Hund, um regelmäßig raus zu kommen, andere arbeiten im Garten. Ich empfehle gerne Tanzen und Bewegung gemeinsam mit Freunden und Bekannten. Vor allen Dingen muss man den Leuten die Angst nehmen, sie müssten nun alles auf einmal machen. Letztlich soll es Spaß und Freude bereiten.

Das Interview führte Thomas Meißner

\section{Chirurgie der Hand}

- Degenerative Erkrankungen werden aufgrund der demografischen Entwicklung weiter zunehmen und entwickeln sich zu einem Top-Thema. Während der Ausbildung lernen Orthopäden und Handchirurgen jedoch nur wenig über Theorie und Praxis der degenerativen Erkrankungen und der rheumatischen Deformitäten der Hand.

Das Buch bietet einen exzellent gegliederten Text, unterstützt durch hervorragendes Bildmaterial. Detaillierte Darstellungen der diversen OP-Techniken werden ergänzt durch zugehöriges Hintergrundwissen mit vielen praxisrelevanten Tipps, die Beschreibung der medikamentösen Therapie und von Orthesen. Auch Schemata zur Nachbehandlung fehlen nicht.

Aus der Praxis für die Praxis beantworten die Autoren detailliert sämtliche Fragen, mit denen sich die handchirurgisch tätigen Ärzte in ihrer täglichen Arbeit konfrontiert sehen.

Das Autorenteam, sämtlich erfahrene Spezialisten, bietet handchirurgischen Anfängern und Profis gleichermaßen ein didaktisch hervorragend aufbereitetes Wissen.

Fazit: Überaus empfehlenswert für alle mit Handchirurgie befassten Orthopäden, Chirurgen und Unfallchirurgen. Der Preis von 199,95 Euro ist der exzellenten Ausstattung des Buches angemessen.

Prof. Klaus A. Milachowski

\section{Praxisbuch Unfallchirurgie}

— Mit seinem Gewicht von 3,7 kg ist das „Praxisbuch Unfallchirurgie" als Bettlektüre zwar denkbar ungeeignet. Dennoch ist dieses in 2. Auflage beim Springer Verlag erschienene Standardwerk wärmstens allen Kollegen zu empfehlen, die unfallchirurgisch tätig sind. Egal ob man die Indikation überprüft, alternative Verfahren sucht oder die einzelnen Schritte einer Operation auffrischen möchte, in allen Fällen findet der Leser eine Fülle praxisrelevanter Informationen.

Das Buch gliedert sich in drei Teile. Im ersten Teil werden, angeordnet nach Körperregionen, die verschiedenen Operationstechniken detailliert und reich bebildert beschrieben. Hierbei soll die Diskussion typischer Gefahren und Komplikationen helfen, Fehler zu vermeiden. Im zweiten Teil werden Frakturen im Kindesalter behandelt sowie Gefäßverletzungen, Infektionen, Polytraumen, Intensivmedizin, Komplikationen und Extremitätendeformitäten. Der dritte Teil des Werks bietet wertvolles Zusatzwissen, das Unfallchirurgen und auch Orthopäden im klinischen Alltag nützlich ist und für das üblicherweise weitere Lehrbücher herangezogen werden müssen. Beschrieben werden beispielsweise klinische Tests, Ultraschalluntersuchungen, Standardzugänge, das chirurgische Handwerk und die Begutachtung.

Fazit: Dieses Standardwerk darf in keiner orthopädischen und unfallchirurgischen Bibliothek fehlen. Das Buch ist sein Geld mehr als Wert.

Prof. Klaus A. Milachowski



Chirurgie der Hand. Rheuma - Arthrose Nervenengpässe; Hrsg.: M. Merle,

S. Rehart; geb.; 446 S.; 1.007 meist farbige

Abb.; 30 Tab.;

ISBN: 978-3-13-148151-1; $199,95 €$

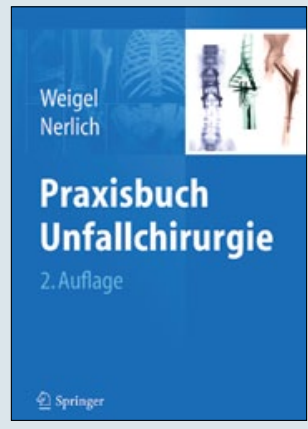

B. Weigel, L. Michael: Praxisbuch Unfallchirurgie; Springer Verlag 2011; 1.500 S.; 3.580 Abb.; 161 Tab.; Hardcover 299,00€; ISBN: 978-3-642-10788-7 